\title{
CHARACTERIZATIONS OF BLOCH FUNCTIONS ON THE UNIT BALL OF $C^{n}$
}

\author{
Dedicated to my father on his 60th birthday
}

\author{
BY Lou ZENGJIAN
}

\begin{abstract}
We give some new characterizations of Bloch functions on the unit ball in $C^{n}$. This extends a theorem of S. B. Lee.
\end{abstract}

\section{Introduction.}

The properties and characterizations of Bloch function on the unit ball have been studied in $[1,2,3,4]$. In this paper we give some new characterizations of Bloch functions, i. e. we give several equivalent conditions for a function to be a Bloch function.

Before we state our main theorem, we fix some notations and definitions used in this paper.

Let $C^{n}$ denote the $n$-dimensional vector space. Let $B_{n}$ denote the open unit ball in $C^{n}$ with boundary $d B_{n}$ and let $a$ denote the rotation-invariant positive measure on $\partial B_{n}$ for which $\sigma\left(\partial B_{n}\right)=1$. Let $U^{n}$ denote the unit polydisk in $C^{n}$, $A\left(U^{n}\right)$ denote the space of all functions which holomorphic in $U^{n}$ and continue on $\bar{U}^{n}$.

Throughout the paper, all the functions we consider are supposed to be holomorphic in $B_{n}$.

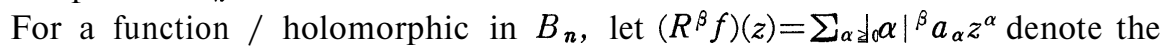
radial derivative of $f(z)=\sum_{\alpha \geqq 0} a_{\alpha} z^{\alpha}$ and $\left(D^{\beta} f\right)(z)=\sum_{\alpha \geqq 0}(|\alpha|+1)^{\beta} a_{\alpha} z^{\text {the }}$ fractional derivative of $f(\beta>0)$. For $0<p<\infty$, we set

$$
M_{p}\left(r, \quad f i=\left(1 / V \int_{\partial B_{n}}|f(r \zeta)|^{p} d \sigma(\zeta)\right)^{1 / p}\right.
$$

where $V$ is the Euclidean volume of $\partial B_{n}$.

DEFINITION 1.1. A holomorphic function $f: B_{n} \rightarrow C$ is said to be in $H^{p}\left(B_{n}\right)$

1980 Mathematics Subject Classification (1985 Revision). Primary 32A10.

Key words and phrases. Bloch function, radial derivative, Hadamard product.

Received November 22, 1991 Revised July 27, 1992. 
$(0<p<\infty)$ if

$$
\|f\|_{H}{ }_{\left(B_{n}\right)}=\lim _{r \rightarrow 1} M_{p}(r, f)<\infty .
$$

DEFINITION 1.2. A function / is said to be in $G^{p}\left(B_{n}\right)(0<p<\infty)$ if

$$
\|f\|_{G^{p}\left(B_{n}\right)}=\left(\int_{0}^{1} M_{1}\left(r, D^{1} f\right)^{p} d r\right)^{1 / p}<\infty .
$$

DEFINITION 1.3. Let $f: \Omega \rightarrow C^{n}$ be an analytic function on the bounded homogeneous domain $\Omega$ in $C^{n}$. For $z \in \Omega$, we set

$$
Q_{f}(z)=\sup \left\{\frac{|\langle(\nabla f)(z), \bar{w}\rangle|}{H_{s}(w, \bar{w})^{1 / 2}}: 0 \neq w \in C^{n}\right\}
$$

where $(\nabla f)(z)=\left(\partial f / \partial z_{1}(z), \cdot \cdot \partial f / \partial z_{n}(z)\right)$ and $H_{s}(w, \bar{w})$ denotes the Bergman metric on $\Omega$ and $\langle u, \bar{v}\rangle$ means $\sum_{i=1}^{n} u_{i} v_{i}$. A holomorphic function on $\Omega$ is called a Bloch function if

$$
\sup \left\{Q_{f}(z): z \in \Omega\right\}<\infty .
$$

The space $B(\Omega)$ of Bloch functions on $\Omega$ forms a Banach space with the Bloch norm $([1])$

$$
\|f\|_{B(\Omega)}=|f(0)|+\sup \left\{Q_{f}(z): z \in \Omega\right\} .
$$

Let $\Omega=U^{n}$. Then $f \in B\left(U^{n}\right)$ if and only if ([1])

$$
\sup \left\{\left|\frac{\partial f}{\partial z_{j}}(z)\right|\left(1-\left|z_{j}\right|^{2}\right): z \in U^{n}\right\}<\infty, \quad 1 \leqq \jmath \leqq n .
$$

Let $\Omega=B_{n}$. Then $f \in B\left(B_{n}\right)$ if and only if ([1])

$$
\sup \left\{|(\nabla f)(z)|(1-|z|): z \in B_{n}\right\}<\infty .
$$

The main result of this paper is the following

Theorem. Let $g$ be a holomorphic function defined on $B_{n}$. Then the following conditions are all equivalent.

(C) The function $g$ is a Bloch function, i.e. $g \in B\left(B_{n}\right)$

(R) $f * g \in A\left(U^{n}\right)$ for all $f \in G^{1}\left(B_{n}\right)$

(C) $f * g \in B\left(U^{n}\right)$ for all $f \in G^{1}\left(B_{n}\right)$

(R) $f * g \in B\left(U^{n}\right)$ for all $f \in H^{1}\left(B_{n}\right)$

where $(f * g)(z)=\sum_{\alpha \geqq 0} a_{\alpha} b_{\alpha} \omega_{\alpha} z^{\alpha}$ iS the Hadamard product of $f(z)-\sum_{\alpha \geq 0} a_{\alpha} z^{\alpha}$ and $g(z)=\Sigma_{\alpha \geqq 0} b_{\alpha} z^{\alpha}$, and

$$
\omega_{\alpha}=\int_{\partial B_{n}}\left|\zeta^{\alpha}\right|^{2} d \sigma(\zeta)=\frac{(n-1) ! \alpha !}{(n-1+|\alpha|) !}
$$

Now let $X$ and $Y$ be two holomorphic function spaces. We let $(X, Y)$ denote the collection of all multipliers from $X$ to $Y$. That is, $(X, Y)$ is the set of all holomorphic function $g$ such that for every $f \in X, f * g \in Y$. 
From Theorem we have

COROLLARY. $\quad B\left(B_{n}\right)=\left(G^{1}\left(B_{n}\right), A\left(U^{n}\right)\right)=\left(G^{1}\left(B_{n}\right), B\left(U^{n}\right)\right)$

$$
=\left(H^{1}\left(B_{n}\right), B\left(U^{n}\right)\right)
$$

This Corollary extends a theorem of S. B. Lee ([7, Theorem 3.6]).

\section{Proof of Theorem.}

At first, it is easy to see that the defining condition (2) of Bloch functions is equivalent to either of the following two conditions

or

$$
\sup \left\{(1-|z|) \mid\left(R^{1} f\right)(z): z \in B_{n}\right\}<\infty
$$

$$
\operatorname{srp}\left\{(1-|z|)\left|\left(D^{1} f\right)(z)\right|: z \in B_{n}\right\}<\infty
$$

We prove the theorem proving the implications $(1 \rightarrow$ (2) $\rightarrow$ (3) $\rightarrow$ (1), (1) $\rightarrow$ (4) and (4) $\rightarrow$ 3. From [5, Theorem 1(b)] we have $G^{1}\left(B_{n}\right) \subset H^{1}\left(B_{n}\right)$. Moreover the proper inclusion $A\left(U^{n}\right) \subset B\left(U^{n}\right)$ )are well known. So the implications (2) $\rightarrow$ (3) and (4) $\rightarrow$ (3) are obvious.

Proof of (1) $\rightarrow$ (2). Suppose $g(z)=\sum_{\alpha \geqq 0} x_{\alpha} z^{\alpha} \in B\left(B_{n}\right)$ and let $f(z)=\sum_{\alpha \geqq 0} a_{\alpha} z^{\alpha}$ $\in G^{1}\left(B_{n}\right), z \in U^{n}$, we have

$$
\int_{\partial B_{n}} D^{1} f(\rho \zeta) D^{1} g(\rho z \zeta) d \sigma(\zeta) \sum_{n=0}(n+1)^{2} \rho^{2 n} \sum_{\alpha \geq 0} a_{\alpha} x_{\alpha} \omega_{\alpha} z^{\alpha}
$$

where $z \bar{\zeta}=\left(z_{1} \bar{\zeta}_{1}, \cdots, z_{n} \bar{\zeta}_{n}\right)$ Since $\int_{j}^{1} \rho^{2 n+1} \log (1 / \rho) d \rho=1 / 4(n+1)^{2}$, we have

$$
4 \int_{\delta}^{1} \rho \log (1 / \rho) \int_{\partial B_{n}} \quad D^{1} f(\rho \zeta) D^{1} g(\rho z \bar{\zeta}) d \sigma(\zeta) d \rho_{\alpha \geq 0}^{\sum} a_{\alpha} x_{\alpha} \omega_{\alpha} z^{\alpha}=(f * g)(z)
$$

By the inequality $\rho \log (1 / \rho) \leqq 1-\rho, 0<\rho \leqq 1$, we have

$$
\begin{aligned}
|(f * g)(z)| & \leqq 4 \int_{0}^{1} \int_{\partial B_{n}}(1-\rho)\left|D^{1} f(\rho \zeta)\right|\left|D^{1} g(\rho z \bar{\zeta})\right| d \sigma(\zeta) d \rho \\
& \leqq 4\|g\|_{B\left(B_{n}\right)} \int_{0}^{1} M_{1}\left(\rho, D^{1} f\right) d \rho \\
& \leqq 4\|g\|_{B\left(B_{n}\right)}\|f\|_{G 1\left(B_{n}\right)}
\end{aligned}
$$

For $f \in G^{1}\left(B_{n}\right), z \in \bar{U}^{n}$, set $f_{z}(\zeta)=f(z \zeta)$. Then, since the correspondence $\bar{U}^{n} \ni z \rightarrow f_{z} \in G^{\mathbf{1}}\left(B_{n}\right)$ is continuous, we have

$$
\begin{aligned}
\mathrm{I} * g\left(z_{1}\right)-f * g\left(z_{2} \mathrm{I}\right. & =\mathrm{I}\left(f_{z_{1}}-f_{z_{2}}\right) * g(e) \mathbf{I} \\
& \leqq C\left\|f_{z_{1}}-f_{z_{2}}\right\|_{G 1\left(B_{n}\right)}\|g\|_{B\left(B_{n}\right)} \\
& \longrightarrow 0 \text { as }\left|z_{1}-z_{2}\right| \longrightarrow 0
\end{aligned}
$$


Hence $f * g \in A\left(U^{n}\right)$ for all $f \in G^{1}\left(B_{n}\right)$.

Proof of (3) $\rightarrow$ (1). For $g(z)=\sum_{\alpha \geqq 0} x_{\alpha} z^{\alpha}$ we define a linear operator $T_{g}: G^{1}\left(B_{n}\right)$ $\rightarrow B\left(U^{n}\right)$ by $T_{g}(f)=f * g$. Then $T_{g}$ is clearly closed, so $T_{g}$ is a bounded linear operator from $G^{1}\left(B_{n}\right)$ to $B\left(U^{n}\right)$. Let

and

$$
f(z)=\frac{\left(1-r^{2}\right)^{n}}{(1-r\langle z, \bar{\zeta}\rangle)^{2 n}}, \quad 0 \leqq r<1, \zeta \in \partial B_{n}
$$

$$
\Psi(z)=\sum_{\alpha \gtrless 0} \frac{\Gamma(|\alpha|+2 n)}{\Gamma(|\alpha|+n)} x_{\alpha} z^{\alpha}
$$

Then we have

and

$$
f(z)=\sum_{\alpha \geq 0} \frac{\Gamma(|\alpha|+2 n)}{\Gamma(2 n) \alpha !}-\zeta^{\alpha} z^{\alpha} r^{|\alpha|}\left(1-r^{2}\right)^{n}
$$

$$
f * g(r e)=\frac{\Gamma(n)}{\Gamma(2 n)}\left(1-r^{2}\right)^{n} \Psi\left(r^{2} \zeta\right),
$$

where $e=(1, \cdots, 1)$. Since $f \in G^{1}\left(B_{n}\right)$ and $T_{g}$ is bounded, there is a constant C independent of $r$ such that

$$
\|f * g\|_{B(U n)} \leqq C\|f\|_{G 1\left(B_{n}\right)}=O(1)
$$

So from (1) we have

Let

$$
\left|\left(R^{1} \Psi\right)\left(r^{2} \zeta\right)\right|=O\left(1-r^{2}\right)^{-(n+1)}
$$

$$
F_{k}(\zeta)=\sum_{|\alpha|=k} x_{\alpha} z^{\alpha}
$$

From (4) we obtain

$$
\left|\sum_{k=0}^{\infty} \frac{k \Gamma(k+2 n)}{\Gamma(k+n)} F_{k}(\zeta) r^{k}\right|=O(1-r)^{-(n+1)}
$$

By [6, Lemma 1] we have

$$
\sum_{k=0}^{\infty} \frac{\Gamma(k+2 n)}{\Gamma(k+n)}\left|F_{k}(\zeta)\right| r^{k}=O(1-r)^{-(n+1 / 2)}
$$

So by the Stirling formula together with (5) and (6) we have

$$
\begin{aligned}
\left|\left(D^{n+1} g\right)(r \zeta)\right|= & \left|\sum_{k=0}^{\infty}(k+1)^{n+1} F_{k}(\zeta) r^{k}\right| \\
\leqq & \left|\sum_{k=0}^{\infty}\left\{(k+1)^{n+1} \frac{\Gamma(k+n)}{k} \Gamma(k+2 n)-1\right\} \frac{k \Gamma(k+2 n)}{\Gamma(k+n)} F_{k}(\zeta) r^{k}\right| \\
& +\left|\sum_{k=0}^{\infty} \frac{k \Gamma(k+2 n)}{\Gamma(k+n)} F_{k}(\zeta) r^{k}\right| \\
= & O(1-r)^{-(n+1)}
\end{aligned}
$$

Hence 


$$
\left|D^{1} g(r \zeta)\right|=O(1-r)^{-1}
$$

From (3) we have $g \in B\left(B_{n}\right)$.

Proof of (1) $\rightarrow$ (4). Let $g \in B\left(B_{n}\right)$, Let / be in $H^{1}\left(B_{n}\right)$ and $z \in U^{n}$. Then

$$
f * g(z)=\int_{\partial B_{n}} f(r \zeta) g\left(r^{-1} z \bar{\zeta}\right) d \sigma(\zeta),
$$

where $\max \left\{\left|z_{j}\right|: 1 \leqq j \leqq n\right\}<r<1$. For $\zeta \in \partial B_{n}$, by [1, Th. 3.4] $G(z)=g(z \bar{\zeta}) \in$ $B\left(U^{n}\right)$, for $j=1, \cdots, n$ and $z \in U^{n}$, we have

$$
\begin{aligned}
\left(1-\left|z_{j}\right|^{2}\right)\left|\frac{\partial(f * g)}{\partial z_{j}}(z)\right| & \leqq \int_{\partial B_{n}}|f(\zeta)|\left(1-\left|z_{j}\right|^{2}\right)\left|-\frac{\partial G}{\partial z_{j}}(z)\right| d \sigma(\zeta) \\
& \leqq C\|f\|_{H^{1}\left(B_{n}\right)}\|G\|_{B\left(U^{n}\right)}
\end{aligned}
$$

Hence we have $f^{*} g \in B\left(U^{n}\right)$. This completes the proof of Theorem.

Acknowledgement. The auther is grateful to Professor Li Qiongyao for her help. He also express his thanks to the referee and the Editor for their valuable and useful comments and suggestions.

\section{REFERENCES}

[1] R.M. Timoney, Bloch functions in several complex variables, I, Bull. London Math. Soc., 12 (1980), 241-267.

[2] R.M. TIMONEY, Bloch functions in several complex variables, II, J. Reine Angew. Math., 319 (1980), 1-22.

[ 3 ] J. A. Cima \& I. GRAHAM, Removable singularities for Bloch and BMO functions, I11. J. Math., 27 (1983), 691-703.

[4] Z.J. Lou, Multipliers of $H^{p}, G^{p}$ and Bloch spaces, Math. Japon., 36 (1993), 21-26.

[5] J.H. SHI, On the rate of growth of the means $M_{p}$ of holomorphic and pluriharmonic functions on bounded symmetric domains of $C^{n}$, J. Math. Anal. Appl., 126 (1987), 161-175.

[6] P.L. Duren, B.M. ROMBERG \& A. L. SHIELDS, Linear functional on $H^{p}$ spaces with $0<p<1$, J. Reine. Math., 238 (1969), 32-60.

[7] S.B. LEE, Multipliers of $G^{p}$ and Bloch functions, Math. Japon., 32 (1987), 421426.

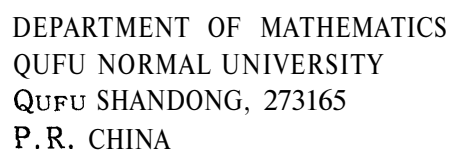

